Monte Carlo Simulation of

Proton-induced Cosimc Ray

Cascades in the Atmosphere

C. A. Hagmann, D. J. Lange, D. M. Wright

March 28, 2007 
This document was prepared as an account of work sponsored by an agency of the United States Government. Neither the United States Government nor the University of California nor any of their employees, makes any warranty, express or implied, or assumes any legal liability or responsibility for the accuracy, completeness, or usefulness of any information, apparatus, product, or process disclosed, or represents that its use would not infringe privately owned rights. Reference herein to any specific commercial product, process, or service by trade name, trademark, manufacturer, or otherwise, does not necessarily constitute or imply its endorsement, recommendation, or favoring by the United States Government or the University of California. The views and opinions of authors expressed herein do not necessarily state or reflect those of the United States Government or the University of California, and shall not be used for advertising or product endorsement purposes.

This work was performed under the auspices of the U.S. Department of Energy by University of California, Lawrence Livermore National Laboratory under Contract W-7405-Eng-48. 
DMC doc 0v1

UCRL-TR-

\title{
Monte Carlo Simulation of Proton-induced Cosmic Ray Cascades in the Atmosphere
}

\author{
Cosmic-ray Physics Team \\ Lawrence Livermore National Laboratory
}

February 15, 2007

\begin{abstract}
We have developed a Monte Carlo model of the Earth's atmosphere and implemented it in three different codes (GEANT4, MCNPX, and FLUKA). Primary protons in the energy range of $1 \mathrm{GeV}-100$ $\mathrm{TeV}$ are injected at the top of the atmosphere. The codes follow the tracks of all relevant secondary particles (neutrons, muons, gammas, electrons, and pions) and tally their fluxes at selectable altitudes. Comparisons with cosmic ray data at sea level show good agreement.
\end{abstract}

\section{Model of Atmosphere}

The atmosphere was modeled as a series of 42 constant-density flat layers, each composed of 78 change between adjacent layers was set to $10 \%$, and the densities were derived from the 1976 US Atmosphere Model. The top of the atmosphere in our model was located at an altitude of $\sim 31 \mathrm{~km}$, and the integrated column density was $\sim 1000 \mathrm{~g} / \mathrm{cm}^{2}$. Void cells were placed at both top and bottom.

\section{Cosmic Primaries}

The flux of cosmic ray particles in the atmosphere and on the ground is mostly due to galactic protons, with minor contributions from alphas and heavier nuclei. At present, we restrict ourselves to the MC modeling of primary protons only. Figure 1 shows the spectrum of galactic protons at Earth. The low-E flux is anticorrelated with the solar wind. As more magnetized solar plasma fills the interplanetary system, the more low-E energy galactic protons are deflected away from Earth. The Earth's magnetic field acts as a further filter on the low-E spectrum, effectively imposing a low energy latitude-dependent cutoff $(\sim 15 \mathrm{GeV}$ at the equator, $0 \mathrm{GeV}$ at the poles). 


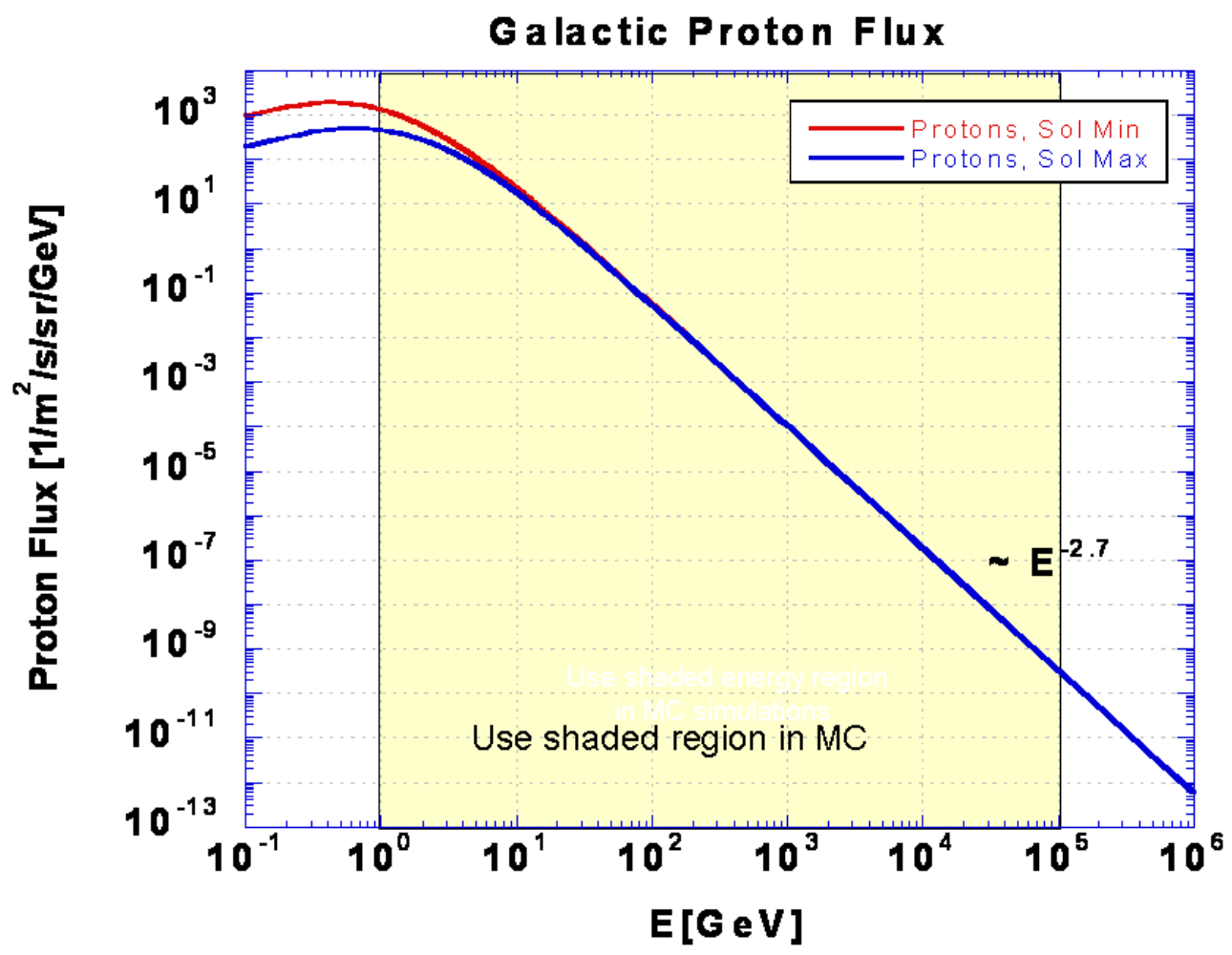

Figure 1: Spectrum of Galactic Protons incident on Earth [1].

\section{Inter-Code Comparisons}

Instead of relying on a single MC code and to guard against potential pitfalls, we ran a couple of problems with three independently developed multi-purpose codes, i.e. Fluka-2005, Geant4, and Mcnpx-v2.5.0. The same model of the atmosphere was implemented in each code and primary protons of fixed energy were injected at the top and the particles of interest (muons, neutrons, protons, gammas, electrons, and pions) were tallied at sea level.

Our general conclusion is that the codes give almost equivalent spectra for all particles except neutrons below $1 \mathrm{MeV}$, where Geant 4 predicts too small of a flux. Neutron transport at these energies has only recently been implemented in Geant 4 . On the other hand, Fluka and especially Mcnpx have been heavily used and are considered verified and validated in this energy region.

Figures 2 and 3 show the tallied neutron and gamma spectra at sea level for incident proton energies of $1 \mathrm{TeV}$ and $100 \mathrm{GeV}$ respectively. 


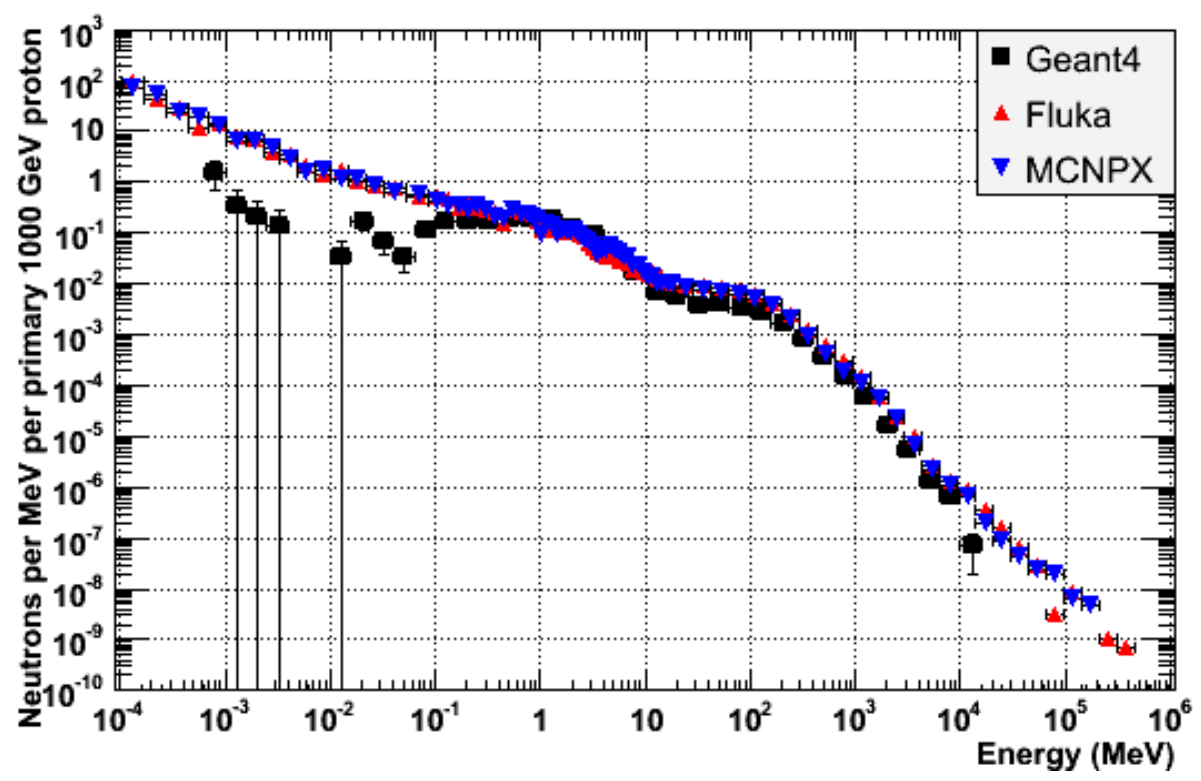

Figure 2: MC-generated neutron spectra at sea level. The incident proton energy is $1 \mathrm{TeV}$.

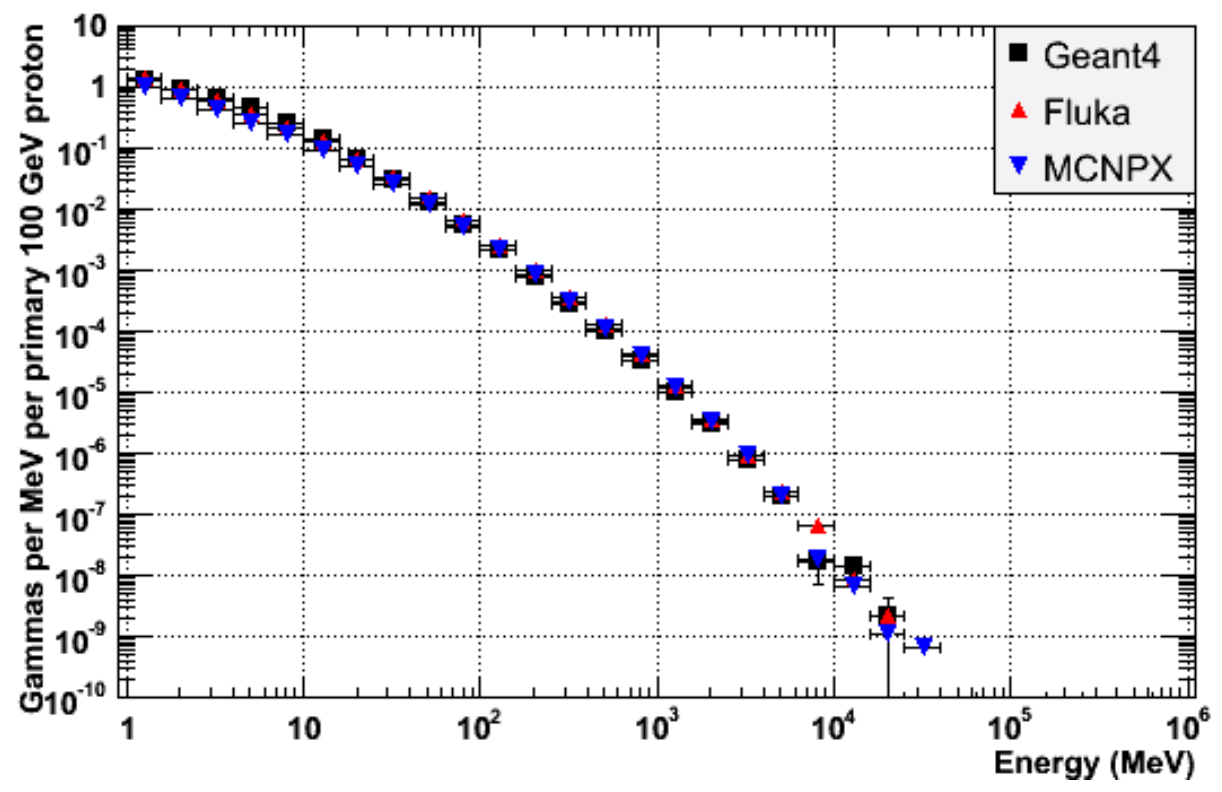

Figure 3: MC-generated gamma spectra at sea level. The incident proton energy is $100 \mathrm{GeV}$. 


\section{Code-Data Comparisons}

Most of our high statistics production runs were made with Mcnpx v.2.5.0 and comprise i 10000 cpu-hrs. To this end, we divided the shaded region into 33 energy groups: 14 equally spaced flat bins from $1 \mathrm{GeV}$ to 15 GeV, plus 19 logarithmically spaced bins up to $100 \mathrm{TeV}$. The latter ones had a power law distribution within each bin with a spectral index of 2.7. All protons were sourced with an isotropic angular distribution and the atmosphere response functions were accumulated for each incident energy bin. By suitable weighting and co-adding of sub spectra, total absolute spectra at any position in the solar cycle and any value of the geomagnetic cutoff can be calculated.

Shown in Figures 4-7 are the predicted muon, proton, pion, and neutron fluxes for no geomagnetic cutoff and average solar modulation, along with the data points from Rastin[2], Brooke and Wolfendale [3], Ashton and Saleh [4], and Kornmayer et al [5] .

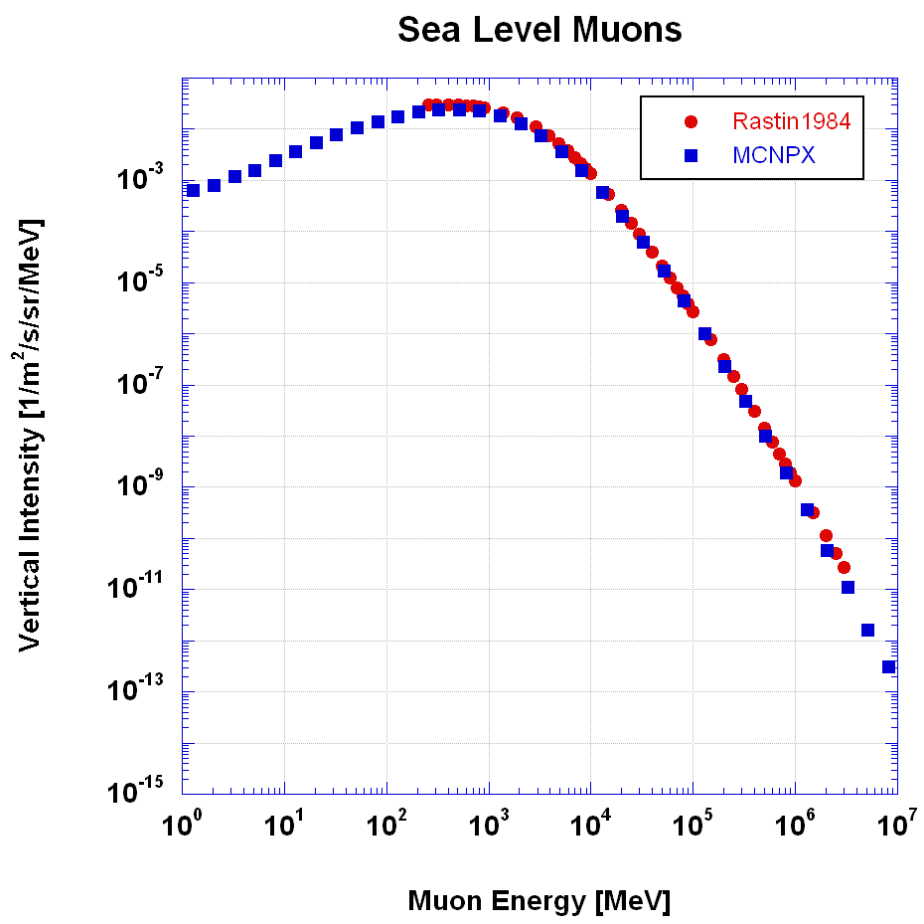

Figure 4: MC-generated muon spectrum and data measured at sea level. 


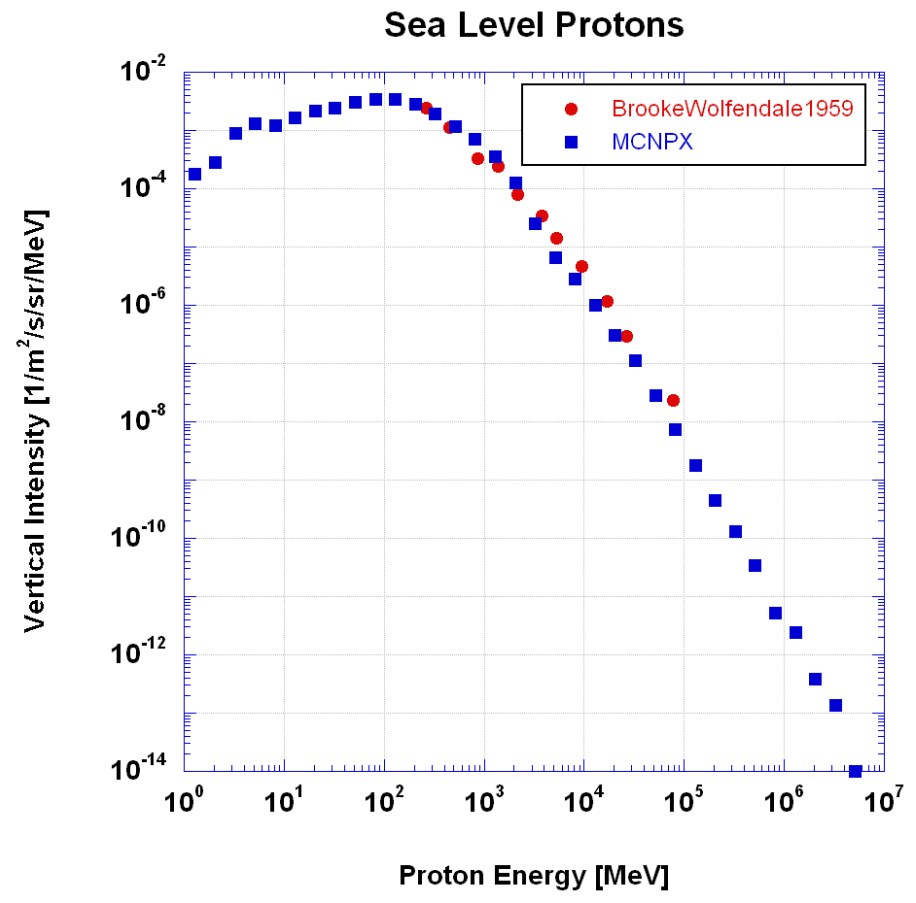

Figure 5: MC-generated proton spectrum and data measured at sea level.

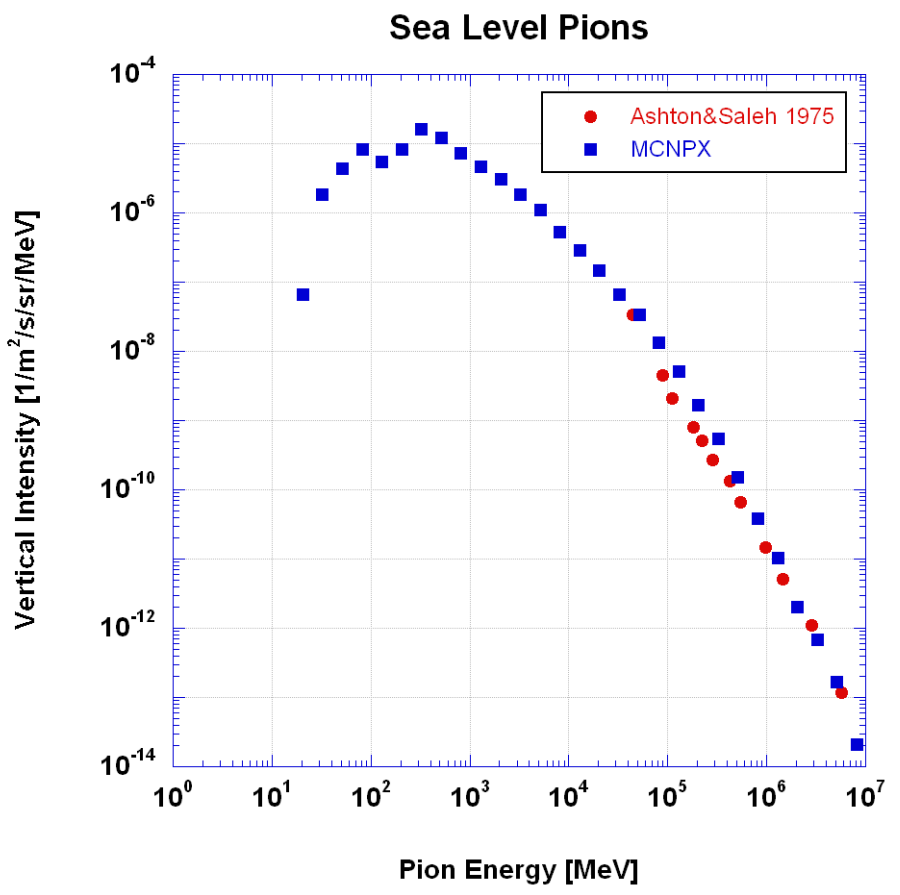

Figure 6: MC-generated pion spectrum and data measured at sea level. 
Sea Level Neutrons

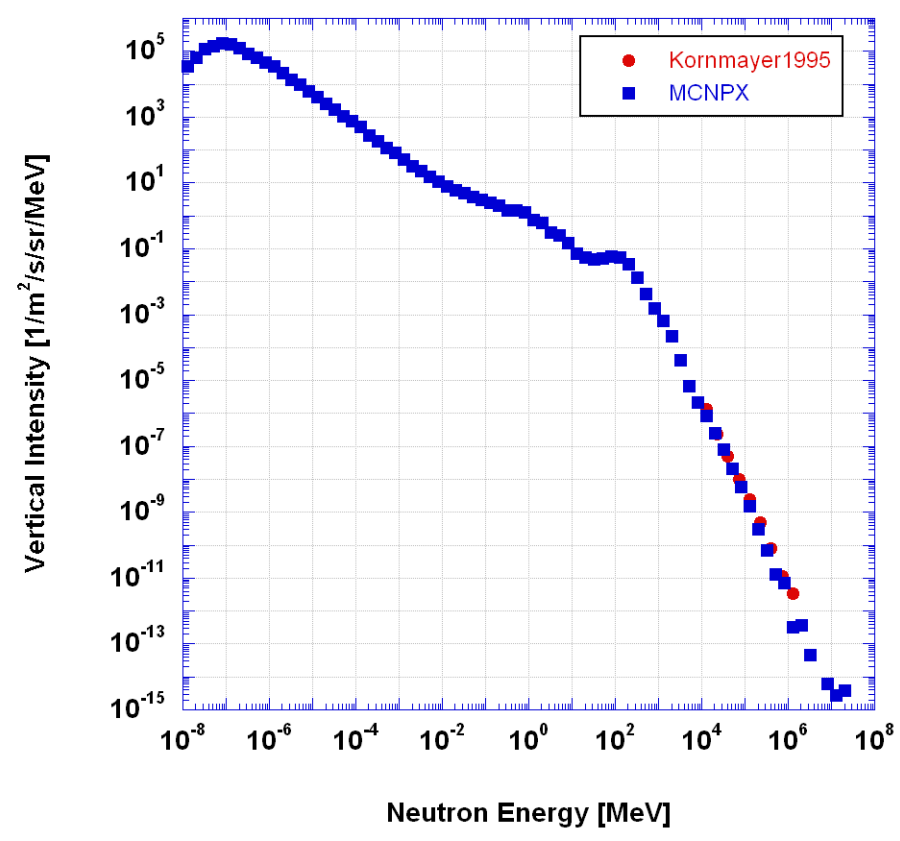

Figure 7: MC-generated neutron spectrum and data measured at sea level. 


\section{Conclusion}

We have calculated cosmic ray fluxes at sea level employing three different Monte Carlo codes. Except for low energy neutrons, all codes give essentially similar distributions of cosmic ray secondaries. We have also compared our calculations to published data and found good agreement. Hence MC calculations should give quite reliable predictons of cosmic ray distributions in situations where data are not available.

\section{References}

[1] P. Papini, C. Grimani, and S.A. Stephens, Nuovo Cim C 19, 367 (1996).

[2] B.C. Rastin, J. Phys. G: Nucl. Part. Phys. 10, 1609 (1984).

[3] G. Brooke and A. Wolfendale Proc. Phys. Soc. 83, 843 (1964).

[4] F.Ashton and A. Saleh PICRC 7, 2707 (1975).

[5] H. Kornmayer et al., J. Phys. G: Nucl. Part. Phys. 21, 439 (1995). 